complex. Meanwhile, the value of the hypothesis is that it widens the practical approach to the separation of solutes on the chromatogram.

We have benefited from discussions with numerous colleagues, but wish particularly to thank Prof. G. E. Briggs for stimulating criticism. We wish to thank Mr. E. T. Whitmore, who assisted with the experi. mental work.

1 Consden, R., Gordon, A. H., and Martin, A. J. P., Biochem. J., 38, 224 (1944).

Consden, R., Nature, 162, 359 (1948).

Cook, A. H., Nature, 164, 300 (1949).

- Burstall, F. H., Davies, G. R., Linstead, R. P., and Wells, R. A., Nature, 163, 64 (1949).

Jermyn, M. A., and Isherwood, F. A., Biochem. J., 44, 402 (1949).

- Martin, A. J. P., and Synge, R. L. M., Biochem. J., 35, 91 (1941).

"Isherwood, F. A., Biochem. J., 40, 297 (1946).

"Moore, S., and Stein, W. H., "Chromatography", Ann. N.Y. Acad. Sci., 49, 265 (1948).

- Berenblum, I.. and Chain, E., Biochem. J., 32, 295 (1938).

${ }^{10} \mathrm{Katz}$, J. R, Kolloid Chem., Beihefte, 9, 1 (1917).

$$
\text { tops }
$$

\section{SOURCE OF THE COSMIC RAYS}

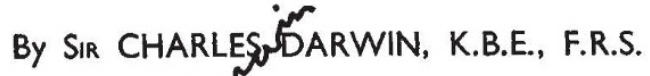<smiles>C1CC2CC3CC1C23</smiles>

$T$ HE enormol energy possessed by the cosmic rays haspiven rise to many speculations as to how they poyld be generated, which have gone deep into the plperties of the ultimate particles, and into the disfripution of matter in outer space ${ }^{1}$. The purpose of the present note is to direct attention to a mâf cruder and simpler mechanism which does not expear to have been considered. In putting it forward I do not intend to imply that it is necessarily exclusive, but only that its potentialities should be examined ; it might provide the whole effect, or it might provide a useful starting point for other mechanisms to work on.

The great developments in recent times of physics in general and of astrophysics in particular have been mainly concerned with static systems, or with systems in nearly steady states. The guiding principle in such work has been the idea of temperature, and this idea has tended to take a chief place in our minds. An example of this arose not long ago, when the sun was found to emit radio noises; though the matter is now in better perspective, there was at first almost a suggestion that because their intensity, if fitted into Planck's formula, implied a million degrees, therefore this must really be the temperature of part of the sun's surface. One might imagine on the same principle that a Martian astronomer, observing through his telescope a thunderstorm on the earth, would recognize that he was seeing electrons with a million electron-volts of energy, and would deduce that the temperature of the earth's surface must really be hundreds of millions of degrees. It is evident that the idea of temperature is sometimes a very poor guide.

When the conditions depart widely from being static, there is no necessary tendency towards equipartition, but the energy may instead become enormously concentrated into certain small parts of the system. Thus in the crack of a whip the tip of the lash is moving faster than the speed of sound, though the coachman's wrist never moves at all fast. Again, when a large sea-wave strikes the wall of a lighthouse, spray is thrown up to a great height, and this in spite of its later rise being much slowed by air resistance. It is not easy to see how to make any general dynamical analysis which would show the circumstances in which such concentrations may occur ; but one example may be given which suggests the almost limitless inequality in the partition of energy that may arise spontaneously in perfectly natural motions.

Early in the century there was much trouble with the destruction of marine propellers by cavitation, and Rayleigh ${ }^{2}$, following the work of others, wrote a paper in which he discussed the collapse of a vacuous bubble in water. Simple hydrodynamic theory leads to the result that the rate of collapse of a spherical bubble is given by the equation :

$$
U^{2}=\frac{2}{3} \frac{p_{\infty}}{\rho}\left(\frac{l^{3}}{R^{3}}-1\right),
$$

where $p_{\infty}$ is the pressure in the remoter parts of the water, $\rho$ its density, $l$ the initial radius of the bubble, $R$ its radius at any time, and $U$ its radial velocity at this time. Suppose now that there is a hemispherical bubble of radius $1 \mathrm{~cm}$. centred on a plane wall, and that at the centre there is a hole of size such as to let through one water molecule. Then $p=1$, $p_{\infty}=10^{\circ}$ for the pressure of the water, $l=1$, and say $R=5 \times 10^{-8}$. This yields $U=7 \times 10^{13} \mathrm{~cm} . / \mathrm{s}$. as the speed with which the molecule will emerge, and it happens in less than a thousandth of a second. If it were legitimate to apply the rules of ordinary dynamics to such a fantastic velocity, the energy of the single molecule would be 80,000 ergs ! It would, of course, be absurd to invoke such an argument literally for the explanation of the cosmic rays, but it does seem worth while to look rather more deeply into the matter, since even if the effect were reduced many millions of times it would still provide ample energy for their generation.

It may be objected that the conditions assumed are very abnormal, in that the bubble would never be perfectly spherical. The solution for the collapse of a non-spherical bubble would be difficult; but a rough estimate may be made of the behaviour of such a bubble by assuming that though it is still spherical, the hole from which the molecule escapes is placed eccentrically. If the point of escape is at $R=10^{-2} \mathrm{~cm}$., then $U=8 \times 10^{5} \mathrm{~cm} . / \mathrm{s}$., a velocity greater than that produced by the detonation of most explosives. Thus, even if a tolerance of one per cent is accepted in the sphericity of the bubble, there is still a very large effect.

More convincing still is the evidence of the pitting of actual propellers by cavitation. Here there seems no reason to expect that the bubbles will be at all accurately spherical, and yet the effect undoubtedly occurs. In some cases propellers have been badly pitted in a single crossing of the Atlantic. Such a voyage might involve a million revolutions of the screws, and at a guess each revolution might give rise to a hundred bubbles. Thus in a hundred million bubbles there are enough of shape sufficiently spherical to produce the effect of high explosive and make many pits in the surface of the propellers. We can see from this that the phenomenon is not even very rare.

An unnatural feature in the calculation is that it assumed the water incompressible. Rayleigh showed, however, that very high pressures are developed just beyond the surface of the bubble, so that the water 
must really be considerably compressed, and the actual occurrence of pitting proves that in any event some compression does not diminish the effect. For a gas the problem of the collapsing bubble becomes immensely more difficult, and to the best of my knowledge it has not been solved. Even the motion in one dimension is formidable. The solution for an advancing wave was given by Poisson $^{3}$ and Earnshaw", and has been fully discussed by Rayleigh ${ }^{5}$. The front of the advancing wave continually steepens, and finally forms a 'bore' with discontinuous velocity at the wave-front, after which no one quite knows what will happen. This bore is obviously an important feature, but its effect must not be over-rated, since it does not involve any increase in the maximum velocity attained. For the spherical motion of the collapsing bubble the solution fails; but it seems certain that pressure and velocity must rise largely with the diminishing area, and it seems likely that the bore will enhance the tendency to concentrate high velocity into the wave-front, and so will encourage the escape of particles of exceptional velocity. There seems no reason why the effect should depend on a very different power of the radius from that found in the incompressible fluid; but this is only a conjecture, and a full solution is much to be desired.

There can be no doubt of the possibility of high concentrations of energy, but the important question is, of course, the frequency with which they will occur, and this seems a matter scarcely amenable to general dynamical treatment. For the cavitation model a statistical law can be given for the energy of the emerging particles. Imagine that samples are collected by means of a fine pipe with mouth opening arbitrarily anywhere inside the bubble. Then a fraction $N=R^{3} / l^{3}$ will have velocity greater than $U$, and so $N$ will be proportional to $1 / E$, where $E$ is the energy. The statistical distribution will therefore obey the law : $d N \sim d E / E^{2}$. The constant of proportionality is independent of the sizes of the bubbles, but depends on the average $p / \rho_{\infty}$ in the surrounding fluid.

This formula, of course, only applies for low velocities, and the model of an incompressible fluid is entirely unsuitable when relativistic effects are important, so that it is impossible to extrapolate it to high velocities. All that can at present be said is that the formula suggests a not very great decrease in number with increasing energy ; and there is no evident reason to foresee any radical change in the statistics, such as would prevent the creation of a considerable number of particles with very high energy indeed.

When a fluid, which is a mixture of different types of particles, is considered, an even more interesting consequence is found. These types will emerge from the pipe at the same velocity, so that instead of equipartition of energy there will be equipartition of velocity. In other words, the energy of a particle will be proportional to its mass, and this rule will continue to hold at relativistic speeds, since there, too, particles of equal velocity have energy proportional to their mass. Consideration of the final.stages of acceleration suggests that this may be too extreme, and all that can be said is that heavy particles should have more energy than light, though perhaps not quite in proportion to their mass. For each individual type there will, of course, be a statistical distribution of the character discussed in the preceding paragraphs.
There does not seem any way of developing a general dynamical thenry of such efferts; but in so far as it is permissible to generalize from this rather slender basis, the following principles suggest themselves :

(1) Since the energies concerned are out of ell proportion to those of temperature equilibrium, the temperature of the source will only be of very minor importance.

(2) There is no practical limit to the amount of energy that may sometimes be concentrated in some of the emitted particles.

(3) At lower energies the statistical law should not be very different from $d E / E^{2}$ for particles all of a type; the power of $E$ might be changed, but the function should not be radically different. It is not clear how to extrapolate to relativistic speeds, but there is no reason to expect that the numbers would become excessively rare.

(4) The energies of particles of different mass should be proportional to their masses, or at any rate should depart widely in this direction from the rule of equipartition. This should also be true at relativistic velocities.

It is suggested that the cosmic rays may originate from some mechanism of this general kind, and though there may be other possibilities, the most obvious source is from the stormy seas that must cover the surfaces of many of the stars. We know that in the case of the sun there is enormous energy in flares and prominences, and long before it is completely dissipated this energy must break down into turbulent motions of great violence, though they soon become too fine in scale to be visible. In such motions there will appear regions of wildly fluctuating pressure, and from some of them there will spurt out spray, which occasionally will have very high velocities indeed. The important question, of course, is the frequency of these occurrences, and though the cavitation model is rather unlike the actuality, it does suggest that they are not excessively rare.

During its escape the spray will be practically neutral in charge, and so will escape most of the radiation-damping which prohibits the rapid acceleration of charged particles; this damping would be a serious difficulty in most mechanisms that might be imagined for the rapid acceleration of the cosmic rays. After getting free of the surface of the star, the atoms will be gradually stripped of their electrons by collisions; but this effect need not be discussed. One important thing that these collisions must do is to filter off the very large number of slower particles which will be thrown up by the spray. However, in practically any theory of the generation of cosmic rays which does not invoke some idea of 'special creation', this filtration will certainly be needed.

It cannot be decided whether the spray theory is sufficient to explain the cosmic rays until much more work has been done on it. If it should not suffice, then it would be profitable to seek some other rapid method for their generation, since the slower the process the greater the difficulty with equipartition. Nevertheless such a slow process as Fermi's ${ }^{1}$ cannot be excluded, and the spray might provide its starting point. Fermi's theory seems to have difficulty over the activation of the heavier atoms, and the present hypothesis might help, in that they would be ex- 
pected to start with energy higher than protons. This feature may, indeed, serve to discriminate between alternative theories.

Much work will evidently be needed in the difficult field of turbulent motion either to prove or disprove the present hypothesis, and it is in the hope of interesting others in it that $J$ have put it forward in this preliminary manner.

2 See, for example, Fermi, Phys. Rev., 75, 1169 (1949).

Phil. Mag., 34, 94 (1917).

- J. l'Ecole Polytech., 7, 319 (1808).

- Phil. Trans., 150, 133 (1860).

- Proc. Roy. Soc., A, 84, 247 (1910).

\section{3 ste}

THE SCIENCE OF TSETSE CONTROL
BY DR. ST. R. S. MORRIS

T SETSE conflol should never be divorced from research, thdugh it may fall into the two categories-long-range and applied. Long-range control is devoted $10^{\circ}$ the extermination of tsetse, whether concerned with the transmission of trypanosomiasis or st. Like pure research, it has great potential value but the problems of human and animal trypanosomiasis often assume dangerous proportions, demanding the immediate application of remedial measures. In this applied work the scientific side tends to be elbowed aside; yet the need is no less and the range considerably wider than in long-range control. For urgency demands that attention be focused on the tsetse, only inasmuch as it is a menace to man and his animals. Each problem must be assessed at its true value in relation to the welfare and activities of the local people and to the economy of the country as a whole. Consideration has to be given to factors governing the transmission and spread of infection. Measures must be appraised and selected to give the greatest degree of relief in the shortest time, and, a point often overlooked, to fit in with the life of the people with the least disturbance and without leaving a burden of costly maintenance.

Such an ideal is not attainable at once, and its attainment may well be delayed rather than hastened if empiricism is allowed to replace the disciplines of scientific technique. The necessity for applied control to produce results demands the checking of every step and process by systematic recording of observations. Much of value is lost through omission to do this. The admirable settlement scheme at Anchau might have afforded much information on applied entomology, but there are no published data, such as the incidence of tsetse and trypanosomiasis before and after control, which might guide the worker elsewhere. Critical analysis of results is necessary for progress; it is equally important for making one's experiences available to others.

\section{Research and Practice in the Gold Coast}

The applied aspect of tsetse control has guided the work of the trypanosomiasis campaign in the Gold Coast since its inception in 1937. The problem was extensive and urgent. The Northern Territories were involved in one corner of a pandemic of sleeping sickness stretching across the Volta Rivers to the Upper Niger, with 30,000 square miles of country showing 5-15 per cent of the populations infected. Two vectors, Glossina palpalis R.D. and $G$. tachinoides West., were responsible, both requiring the evergreen fringing forest of rivers and streams as permanent habitat and therefore strictly riverine in their distribution. This brought a further complication by causing depopulation along the Black Volta and its larger tributaries, where the disease had long been severe, and overcrowding on the watersheds, with resulting land hunger and erosion.

A problem of such magnitude and far-reaching effects required drastic measures for its control, and this demanded research. Meanwhile, the urgency of the situation was met by mass treatment of patients, and a system of protective clearings which aimed at excluding tsetse from the main points of contact with man, water-holes and river crossings, but which left the greater part of the fly-belt untouched. This effected substantial reductions in the amount of sleeping sickness (60-80 per cent in five years), but the decline was not progressive. However, valuable information was gained. Standardized records of the incidence of tsetse in clearings and in adjacent flybelt and of the sleeping sickness-rate in the localities concerned were kept for three districts, the area of observation totalling 3,000 square miles. A positive correlation was found between the length of clearing and the reduction in tsetse incidence, and between the amount of clearing done in an area and the general fall in the infection-rate. In other words, we were enabled to know exactly what we were doing, to predict the results of various applications of clearing and, consequently, to select methods appropriate to each problem 1 .

This information explained the inadequacy of small-scale measures for the control of serious epidemic trypanosomiasis. First, $G$. palpalis and $G$. tachinoides move freely along open river banks at all seasons, so that clearings even three to five miles in length are traversed regularly by small numbers of flies (this is also experienced with $G$. palpalis in Kenya), half-mile clearings are entered by as much as 25 per cent of the adjacent fly-belt population, and clearings of 200 yards or less may cause an increased incidence of tsetse at the point they are designed to protect. Secondly, localized small clearings do not embrace all the points of man-fly contact where transmission of infection occurs. Thirdly, for small clearings to be effective, all the woody vegetation should be cut; this is expensive and may result in river-bank erosion and silting of water-holes. Finally, small clearings cannot control animal trypanosomiasis nor correct the evils arising from the maldistribution of the population.

Research gave a method for eradication by exploit. ing the habit of these tsetse communities of spreading extensively along the rivers during the favourable climatic conditions of the rains, but contracting to very limited and well-defined foci during the adverse period of the dry season. The tsetse communities are most vulnerable at the time and location of their concentrations. A study of the plant associations of the dry-season habitats was started in $1929^{2}$, con. tinued by J. L. Stewart on the Nabogo River during $1932-36^{3}$, and extended during the present investiga. tions to cover the whole inland savanna zone of the Gold Coast. It was found that these concentration sites were characterized by the presence of certain species of trees and shrubs, limited in number, and that the list of these 'indicators' could be used as a consistent standard for clearing. The argument was 